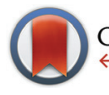

CrossMark

\& click for updates

Cite this: Food Funct., 2016, 7, 20

\title{
Edible oil structuring: an overview and recent updates
}

\begin{abstract}
Ashok R. Patel* and Koen Dewettinck
In recent years, research dealing with edible oil structuring has received considerable interest from scientific community working in the area of food formulation. Much of this interest is linked to the possibility of using structured oil in development of newer product formats with improved nutritional profile (trans fatfree, low in saturated fats and high in mono and/or poly unsaturated fatty acids). In addition to the obvious industrial need of finding the alternative formulation approach, the interesting properties of structured systems (particularly, oleogels) also makes them a fascinating subject for fundamental studies. In this paper, we attempt to give a comprehensive and concise overview of the field of oil structuring with special emphasis on the updates from recent years. Specifically, several categories of food-grade oleogelators and their potential food applications are summarized with typical examples along with a discussion on the general principles and unresolved challenges related to this emerging area.
\end{abstract}

Received 19th August 2015, Accepted 19th September 2015

DOI: $10.1039 / \mathrm{c} 5 f \circ 01006 \mathrm{c}$

www.rsc.org/foodfunction

\section{Introduction}

Nutritional recommendations regarding the consumption of dietary fats have seen many revisions over the years. However, dietary guidelines from national and international health organizations have consistently emphasized the need to lower

Vandemoortele Centre 'Lipid Science \& Technology', Lab of Food Tech \& Engg, Faculty of Bioscience Engg., Ghent University, Coupure Links 653, 9000 Gent, Belgium.E-mail: patel.ashok@ugent.be

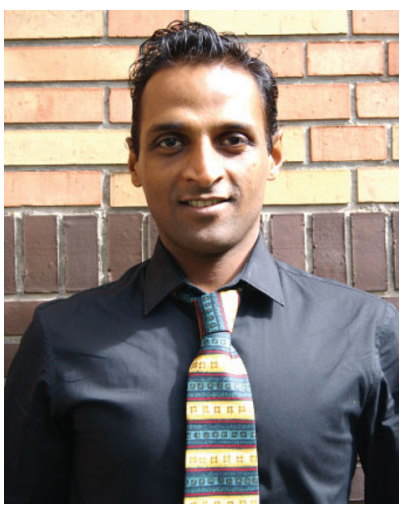

Ashok R. Patel
Ashok Patel earned his PhD degree in Pharmaceutics from Institute of Chemical Technology, India in 2008. Following his postdoctoral research in the area of colloidal delivery systems at Unilever R\&D Vlaardingen as a Marie Curie International Incoming Fellow, he joined Ghent University where he is now an associate professor and research director. His current research (supported by Marie Curie Career Integration Grant) deals with the development of structured oil systems including oleogels and complex food emulsions. He has over 50 publications in the area of applied colloid science including original papers, reviews, book chapters, patents and a book. the consumption levels of trans and saturated fats. The negative effect of trans and saturated fats on cardiovascular health is linked to their role in increasing the levels of LDL (low density lipoprotein) also known as "bad cholesterol", in contrast, mono and polyunsaturated fats tend to lower LDL levels, which is why the nutritional guidelines recommend replacing the trans and saturated fats in diet with unsaturated fats. Most European countries have already placed strict legislative limits (that more or less corresponds to a virtual ban) on trans-fats for quite some years ${ }^{1,2}$ and recently, the US Food and Drug

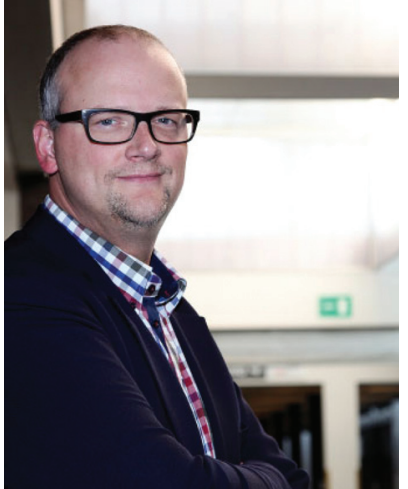

Koen Dewettinck
Prof. Koen Dewettinck is head of the Laboratory of Food Technology and Engineering since 1999 and since 2014 chairing the Department of Food Safety and Food Quality of Ghent University. His research group is involved in food design on a mesoscopic scale. Doing so he wants to answer the consumer requirements for innovative products that are tasty, healthy, convenient and affordable. The main product groups of interest are dairy products, margarines and shortenings, and chocolate and confectionary products. 
Administration (FDA) has also banned the use of partially hydrogenated oils (the main source of artificial trans-fats) in processed foods, the food manufacturers have three years from now to rework the formulation of their products for complete removal of trans-fats. It has been argued recently, that unlike trans-fats, saturated fats are not as bad as they are perceived to be and that substituting saturated fat with carbohydrates (in an attempt to lower saturated fat intake) is actually a worse option. ${ }^{3}$ However, the results of meta-analysis does show that there is a clear benefit of replacing saturated fat with polyunsaturated fat and essential fatty acids. Hence, the dietary guidelines with relation to saturated fat (the current consumption limit is less than $10 \%$ of total calories) is unlikely to change in the near future. Therefore, food manufacturers are currently looking for ways to formulate their food products without the use of significant amount of solid fats. However, formulating food products in absence of solid fats is quite challenging as they are responsible for providing the required structure, texture, and mouth feel to the products.

Among different approaches that can be explored for trans and saturated fat replacement, oleogelation (converting liquid oil into gel-like material without modifying the chemical characteristics of oil) has been hailed as a feasible approach that can help us address this challenge. The possibility of gelling $\geq 90 \mathrm{wt} \%$ of liquid oil at a relatively lower mass fraction of gelator molecules makes oleogelation a very efficient means to improve the nutritional profile of food products (trans-fat free, low in saturated fats and high in unsaturated fats). This efficient structuring in oleogels is typically achieved by supramolecular assemblies (building blocks) of gelator molecules that organize into a 3-D network which can trap a large amount of oil into a gel-like structure.

\section{Oleogelation: background}

As a research domain, oleogelation for food structuring is still in its infancy but it has already received a great deal of interest from academic researchers and industrial scientists alike. Structured oil systems formed via oleogelation are known by the names of oleogels, organogels, lipid gels or simply, oil gels. Historically, the gelation of liquid oils into self-standing, structured solids (gels) have been studied for many years by researchers from both non-bio related (analytical chemistry, material science) and bio related fields (drug delivery and cosmetics). For instance, a simple search on Web of Science ${ }^{\mathrm{TM}}$ with key words 'oleogel' or 'organogel' gives more than 2000 hits with first reference to word 'oleogels' dating back to early 50 s where the properties of oil gels were studied for drug delivery and cosmetic applications. In contrast, using oleogelation for food structuring purposes is relatively recent phenomenon which has seen a proliferative growth only in the last few years (Fig. 1).

Since the publication of first results in $2003,{ }^{4}$ more than half-a-dozen of review papers, few book chapters and two books have been published to summarize the research results

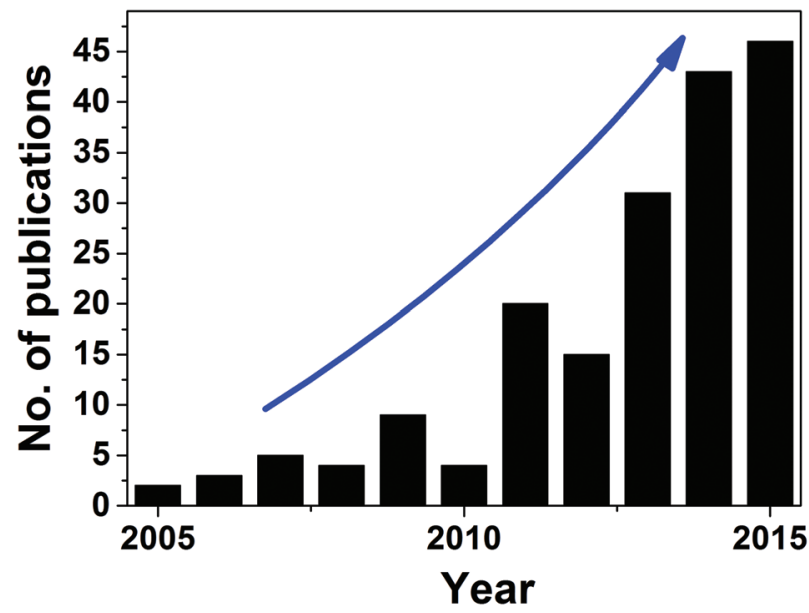

Fig. 1 Number of papers published in the area of edible oil gelation in last 10 years.

in this area. The initial studies dealt mostly with the use of lipidic additives (such as long chain fatty acids, fatty alcohols, dicarboxylic acids, wax esters, hydroxylated fatty acids, natural waxes and partial glycerides) to gel edible oils via direct dispersion at elevated temperatures followed by cooling. ${ }^{4-8}$ This was followed by more explorative research in identifying twocomponent mixtures that showed synergistic gelling functionality such as stearic acid + stearyl alcohol, ${ }^{9} \beta$-sitosterol + oryzanol, ${ }^{10}$ lecithin + sorbitan tristearate $^{11}$ and mixtures of ceramides. ${ }^{12}$ Structured systems designed using polymers such as modified cellulose (ethyl cellulose, EC) ${ }^{13}$ and proteins ${ }^{14}$ were also studied to extend the structuring principle beyond the crystalline network formation as seen with the lipid-based materials. In recent years, research on hydrophilic polymerbased gels, ${ }^{15-17}$ resin wax (shellac wax) gels ${ }^{18,19}$ and inorganic particle-based gels ${ }^{20}$ have also been published. The earlier studies in the field of oleogelation were mainly focused on the fundamental understanding of the structuring principles, but in recent years, reports on potential food applications have also been published. In the following sections, an overview of the approaches used for oil structuring and the potential food applications of structured systems are elaborated using recent examples.

\section{Various approaches for oil structuring}

There are different ways of categorizing oleogelation and oil structuring approaches, for e.g., based on the molecular characteristics of gelators (low molecular weight organic compounds, polymeric compounds and inorganic compounds), ${ }^{21,22}$ chemical type of gelators (lipid and non-lipid based oleogelators), ${ }^{23,24}$ number of gelators used (mono component and mixed system gels) ${ }^{21,25}$ and last but not least, the type of building blocks and structuring principles involved in gelation (crystalline particle and network formation, 


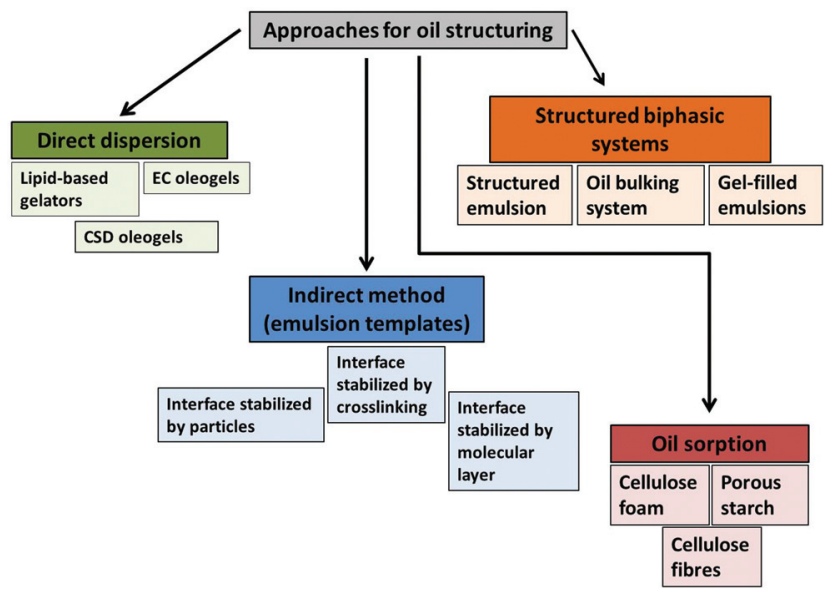

Fig. 2 Various approaches used for edible oil structuring. EC and CSD $=$ ethyl cellulose and colloidal silicon dioxide respectively.

self-assembly induced crystalline or non-crystalline network, particle-filled network and polymeric strands). ${ }^{26}$ In this review, we would like to discuss the structuring approaches by categorizing them based on the processing steps involved in creating the structured oil systems (Fig. 2). To make it more broad, we have also included certain structured biphasic systems (oil bulking systems, structured emulsions, gel-filled emulsions etc.) which cannot be categorized as oleogels per se.

\section{Direct dispersion of structuring agents in oil phase}

Lipid-based gelators such as waxes, fatty acids, fatty alcohols, mono glycerides (MAGs) etc. are usually directly dispersed into oil phase at temperatures above their melting points followed by cooling to lower temperatures either under shear or quiescent conditions. This is the most commonly used process for creating oleogels and as such, it is an extension of conventional structuring process involving solid fats (high melting triacylglycerol molecules, TAGs). Steps of nucleation, crystal growth, aggregation and network formation as seen with solid fats are also involved in oil structuring with lipid-based gelators. However, the type and the morphology of crystals formed, the tendency of their unidirectional growth and lower aggregation among the formed crystals differentiates them from the conventional solid fat crystals, resulting in network formation (that shows high oil binding capacity) at much lower mass fraction of crystalline phase (Fig. 3). For monocomponent gels, such as those prepared using fatty acids and wax esters, the gelation efficiency increases linearly with their acyl chain lengths suggesting a head-to-tail alignment of the linear structures which may lead to irregular network formation stabilized by inter-molecular hydrogen bonding. ${ }^{4}$ For mixed system gels, particularly those prepared by combining lipid-based materials with slight difference in polarity (fatty acids + fatty alcohol and mixtures of lecithin with either sorbitan tristearate or sitosterol), the gelation is influenced by changes in microstructure induced by heterogeneous nucleation, higher crystallization

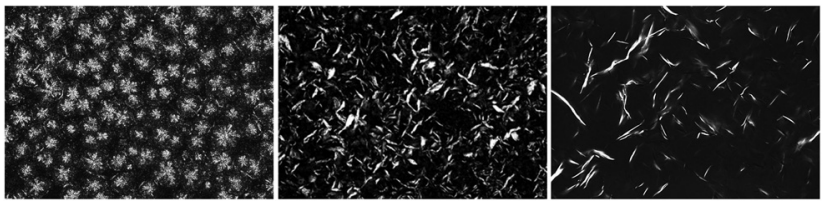

Fig. 3 From left to right: PLM images showing the morphology of crystals formed by high melting TAGs, MAGs and sunflower wax respectively (image width $\approx 800 \mu \mathrm{m}$ ). The crystals from TAGs grow in all dimensions giving rise to almost spherical shape while crystals from lipid additives MAGs and sunflower wax show $1 D$ and $2 D$ growth resulting in more linear shape that aids in the formation of a continuous network at significantly lower crystalline phase.

kinetics (increased nucleation rate due to surface tension reduction), crystal habit modification and network strengthening. ${ }^{6,11,27}$ The influence of mixed components on gelling efficiency is also quite evident in wax-based oleogels since most natural waxes are usually composed of wax esters, $n$-alkanes, fatty alcohols and free fatty acids in varying proportions. As reported recently, the relative proportion of individual components have much higher influence on the gel properties compared to the melting range of waxes. ${ }^{28,29}$ In specific cases, such as hydroxylated fatty acids, ${ }^{7} \beta$-sitosterol + oryzanol $^{30}$ and lecithin + tocopherol, ${ }^{31}$ complex supramolecular structures such as self-assembled fibrillar networks (SAFINs), tubules and cylindrical micelles are responsible for providing structural framework to support gelled structure.

Among non-lipid-based oleogelators, EC (polymer) and colloidal silicon dioxide, CSD (inorganic particle) can also be used for oleogelation through direct dispersion. However, there are certain conditions that require special attention during processing of this gels. For EC gels, the dispersion of polymer in oil is achieved by heating the dispersion above the glass transition temperature of polymer $\left(\approx 140{ }^{\circ} \mathrm{C}\right)$ and shearing at this hold temperature for uniform dispersion. ${ }^{32}$ On cooling below a certain set point, gelation occurs due to the association of polymeric chains mediated via hydrogen bonding. ${ }^{33}$ In some cases, other polymers such as chitin have also been used for creating oleogels by direct dispersion. However, converting native chitin into structuring units like nanocrystals and whiskers through chemical modification (hydrolysis) is a prerequisite. ${ }^{34,35}$ A strong gel using crude chitin is possible only if surfactant (phospholipid or Span 60) is incorporated at $2: 1$ chitin : surfactant ratio. ${ }^{34}$ The surfactant helps in preventing the aggregation of crude chitin in oil solvent leading to a subsequent network formation that can trap liquid oil into a gel structure.

For CSD gels, the dispersion is carried out at room temperature but a high shear $(>10000 \mathrm{rpm})$ is required to de-agglomerate aggregates of primary particles. On removal of shear, the uniformly dispersed aggregates re-agglomerate to form a continuous network which results in the formation of gels which are substantially hard (Elastic modulus, $G^{\prime} \approx$ $\left.10^{4} \mathrm{~Pa}\right) .^{20}$ 
Indirect method using water continuous emulsions as templates

The concept of using a polymer as a structurant for gelling vegetable oils was first introduced way back in $1990 \mathrm{~s}^{36,37}$ The polymer used for this study was EC, a hydrophobic cellulose derivative and till date, it is the only food-grade polymer that can be used for oil gelation through direct dispersion. Using polymers for oil structuring appears promising because there are many polymers that are approved for use in foods and most of them have been well-characterized. However, since, most of the food polymers are inherently hydrophilic in nature, they show limited dispersion in oil (a hydrophobic solvent), rendering them ineffective in structuring oils.

Functionality of polymers to form structural framework in aqueous solvent is well-known and is attributed to their hydration into extended and open conformations that leads to stronger molecular interactions with the solvent (polymersolvent interactions). The hydrodynamic volumes (excluded volumes) of hydrated polymer coils can overlap and subsequently form physical crosslinks (junction zones) at certain concentration $\left(C_{\mathrm{g}}\right)$, resulting in the gelation of water phase. In order to use hydrophilic polymers for oil structuring, it is important to first pre-hydrate them in aqueous phase and arrest these hydrated conformations in dehydrated form such that they can be used for physical entrapment of liquid oils. Some food polymers such as proteins and modified polysaccharides are surface active and conformational framework of these components can be created from their aqueous dispersions by first promoting their adsorption to oil-water interfaces followed by removal of water to obtain dried microstructures with entrapped/embedded oil phase. A range of structured oil systems such as oil powders, gels and soft solids can be created using water continuous emulsions as templates. ${ }^{16,38-40}$ Representative pictures of soft solids (containing more than 97 wt\% liquid sunflower oil) prepared by drying concentrated oil-in-water emulsions are shown in Fig. 4. Due to its unique microstructure (discussed elsewhere), ${ }^{15,16}$ the soft solid is capable of encasing a large amount of liquid oil without showing any oil leakage over extended period of storage. These indirect methods of creating structured oil involves a multistep process including the formation and stabilization of concentrated oil-in-water emulsion, stiffening of adsorbed interfacial layer through crosslinking or complex formation, drying of water phase and mild shearing of dried solids to obtain gels. The success of this approach is dependent on forming a stable interfacial layer that is stiff enough to withstand the dehydration during drying. This has been achieved through three different ways: (a) by stabilizing interfaces using surface active proteins and subsequently crosslinking it through thermal or chemical means, ${ }^{14}$ (b) using protein particles followed by complexation with lipid additive ${ }^{41}$ and (c) using a combination of surface and non-surface active food polymers that can increase the viscoelasticity of the interface. $^{24}$

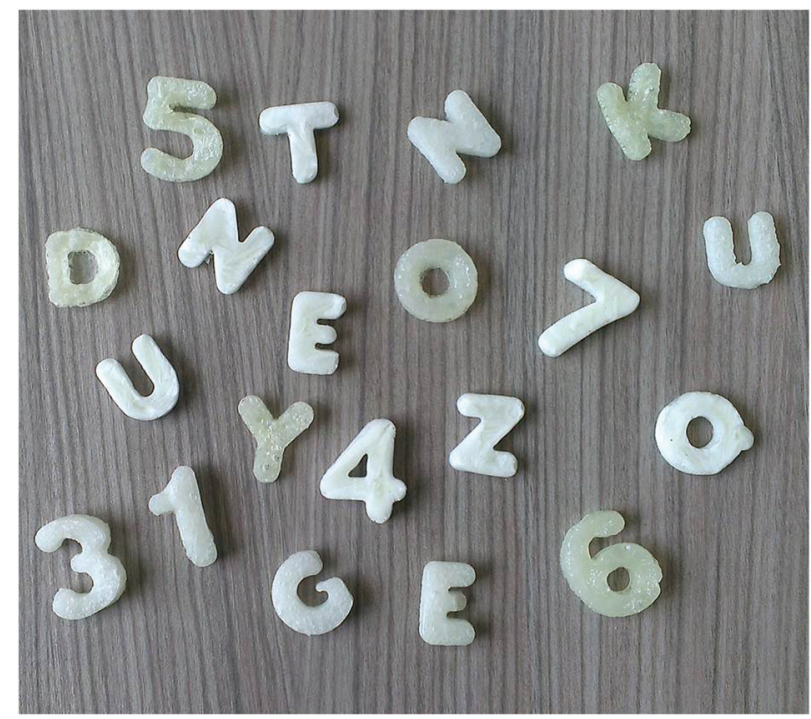

Fig. 4 Soft solids (containing more than 97 wt\% liquid sunflower oil) prepared by drying emulsion templates.

\section{Structuring aided by physical sorption of liquid oil}

Sorption (adsorption and absorption) process can be defined as the enrichment of a material or an increase in the density of a fluid in the vicinity of an interface. ${ }^{42}$ Sorption techniques find use in a range of different fields including catalysis, pollution control, solid state chemistry etc. In foods, porous additives with high specific surface area and other absorbent fillers are usually included in the formulation to accumulate and confine the excess water/moisture in order to increase the consistency, flowability and texture of different product formats. The use of absorbents for edible oil uptake has been rarely explored. In last few years, the issue of oil spills have been constantly in news and consequently there has been a rising interest in developing porous materials (aerogels, hydrogels and cryogels) that can preferentially take up a large quantity of hydrophobic oil in presence of water. Related concept of using porous materials made from food-grade components for oil structuring could result in many interesting applications. Recently, we were successful in creating a porous cellular structure using aqueous foams (stabilized by hydroxyl propyl methylcellulose) as templates. ${ }^{17}$ Due to its high porosity, the porous cryogel could absorb oil at more than 100 times its own weight and subsequently shearing of this oil-sorbed material resulted in the formation of oleogel containing as high as 98 wt\% liquid oil. The structured oil system showed rheological properties corresponding to a strong gel (elastic moduli higher than viscous moduli by a factor of 10 in the linear response region and almost a non-frequency dependent moduli response) but had a weak thixotropic structure recovery properties. However, it was later shown that the thixotropic properties could be improved significantly by incorporating a small amount of hard stock (5 wt\%). ${ }^{43,44}$

The oil absorbing properties of cellulose fibres have also been exploited to formulate shortening compositions with 
lower levels of saturated and trans-fats. ${ }^{45}$ The ability of fibrous cellulose material with capillaries to take-up oil and strengthen the network of crystalline fat were considered important for obtaining such structured systems. Likewise, porous starch particles have also been used to develop structured oil system for bakery applications. ${ }^{46}$ The micron size starch particles $(\leq 20 \mu \mathrm{m})$ with high specific surface area $\left(0.5-1.2 \mathrm{~m}^{2} \mathrm{~g}^{-1}\right)$ and low density $\left(0.3-0.5 \mathrm{~g} \mathrm{~cm}^{-3}\right)$ were created using partial enzymatic degradation.

\section{Structured biphasic systems}

There is a substantial amount of water in most lipid-based food products, for e.g., table spreads which could contain as much as $70-80 \mathrm{wt} \%$ of water. The water-oil biphasic systems, that display 'gel-like' viscoelastic behaviour, can be broadly categorized into three main classes: (i) water continuous emulsions structured using biopolymers (proteins and/or polysaccharides) that act as emulsifiers as well as thickening/gelling agents (e.g. cheese, yogurt, dairy-based desserts etc.); (ii) highly concentrated water continuous emulsions where the structuring is a result of closely crowded dispersed droplets at high packing fractions (such as mayonnaise, sauce etc.) and (iii) oil continuous emulsions structured using crystalline network of fat particles (e.g. margarine, butter and spreads). Among these three classes, the products based on oil continuous emulsions could benefit the most from oil structuring approaches as solid fat plays a significant role in texturing of these products. The most straightforward approach for structuring such products involves gelation/thickening of water phase to add body to the emulsion while decreasing solid fat and/or total fat content of the product. In fact, most commercial low-fat spreads are formulated through this approach by thickening/ gelling of dispersed water droplets with one of the following: (i) viscosity enhancing agents (e.g. milk proteins, starch etc.); (ii) gelling agents (hydrocolloids like alginates, gelatin etc.); (iii) phase-separating mixtures (with thermodynamically incompatible hydrocolloids) and (iv) synergistic combinations (mixtures of hydrocolloids that show synergistic interactions e.g. galactomannans and carrageenans). ${ }^{47}$ However, low-fat spreads still have certain amount of solid fat that contributes to the required functional properties.

Some structured biphasic systems have been created without the use of any solid fat and these systems could be used as baking fats ${ }^{48}$ and for meat processing. ${ }^{49}$ Of these, Coasun SE, a structured emulsion (stabilized by interfacial accumulation of hydrated multilayers of saturated monoacylglycerols, MAGs) is even commercially available for bakery and other applications. ${ }^{48,50}$ This structured emulsion is oil-in-water type and contains almost $30-40$ wt\% water which is sandwiched between the MAG layers, upon crystallization of MAGs, the dispersed oil droplets become encapsulated within the hydrated layers resulting in a semi-solid product with high viscosity. ${ }^{51}$ The structuring is induced by the high volume fraction of dispersed encapsulated oil droplets resulting in a nonflowing but non-self-standing gels.
Other alternative way to create a structured biphasic system is to disperse and physically trap oil droplets in a hydrogel matrix (aqueous phase gelled using hydrocolloids) resulting in the formation of oil-bulking systems. ${ }^{49}$ Hydrocolloids such as Konjac glucomannan and alginates in combination with inulin and dextrin have been tried for structuring systems containing healthy oils such as fish, flaxseed and olive oil. ${ }^{52,53}$ The technology involves the dispersion of liquid oil in aqueous phase followed by the gelation of water continuous phase. The oil-bulking systems are different from conventional water emulsions as no emulsifiers are used for their preparation, the stabilization of system is entirely due to the physical entrapment of oil phase in gelled matrix of water phase.

Attempts have also been made to generate oil continuous structured emulsions where the dispersed water phase is gelled to obtain what is called as 'gel-filled emulsions'. These are different from the above two approaches which could be categorized as 'emulsion-filled gels'. These gel-filled emulsions have been made at different water content from 50 to $90 \mathrm{wt} \% .^{54-56}$ The technique used for making these emulsions involves dispersion of water droplets in oil continuous medium followed by gelation induced either by temperature drop or by polymer-cation interactions.

The three different type of structured biphasic systems described above are shown using simple schematics in Fig. 5. In case of structured emulsions, the interfaces around the dispersed oil droplets are structured with the help of waterswollen, crystallized MAG layers. In oil bulking systems the dispersed oil droplets are embedded in the matrix of water phase gelled using network of polymers and the gelled emulsions are formed by crowded packing of dispersed water droplets gelled using synergistic combination of locust bean gum and carrageenan.

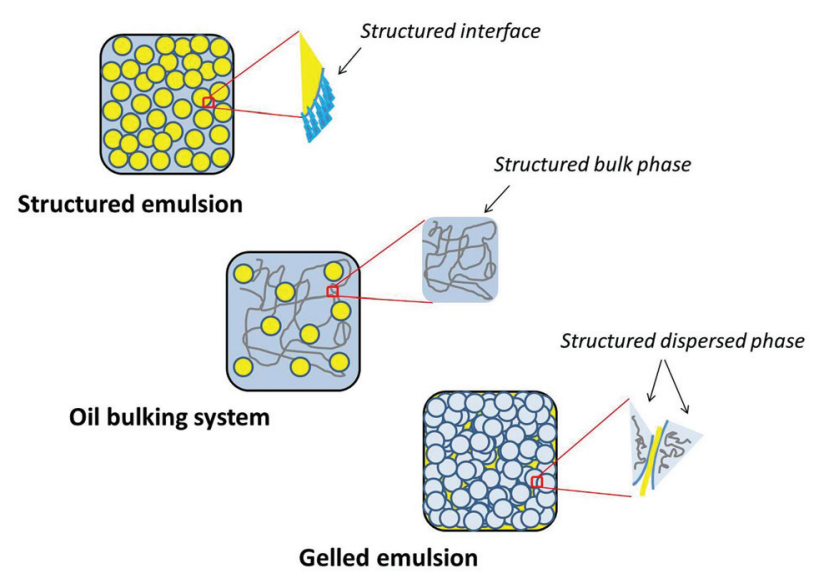

Fig. 5 Schematic representation of three different types of structured biphasic systems: structured emulsions, oil bulking system and gelled emulsion with structured interface, bulk phase and dispersed phase respectively. Structured emulsion and oil bulking system have oil droplets dispersed in water continuous phase whereas the gelled emulsion is composed of tightly packed gelled water droplets dispersed in oil continuous phase. 


\section{Food applications of structured oil systems}

The main functional role of fat in food formulation is to provide structure to the product, the structure consequently influences the textural (hardness, spreadability etc.) and organoleptic properties (melt-in-mouth effect, cooling effects, emulsion break-up and flavour release etc.) in finished products and stabilization (enhancing air incorporation, preventing gluten network formation etc.) in products meant for intermediate use, such as baking fats. ${ }^{57}$ Other functionalities that are linked to the structure may also include physical entrapment of low molecular weight bioactives (for controlled delivery of nutraceuticals) and controlling oil mobility and migration (for imparting stability to chocolate products). ${ }^{58}$

The applications of structured oil systems in four different product categories are summarized and discussed below.

\section{Baking fats}

Different types of fats are used for bakery products such as bread, biscuits, pastries and cakes. Irrespective of the product type, shortening and butter (or baking margarine) are the two most commonly used classes of bakery fats. The functional roles of baking fats are manifold and quite complex. Specifically, the structured fat breaks the continuity of protein (gluten) and starch network in the batter or dough which results in a tender, non-chewy texture of baked products. Structured fat also provide a thin lubricating film over tiny solid particles in bakery mass, thus imparting long-lasting softness and freshness to finished products. In addition, they further contribute to increased incorporation and stabilization of finer air bubbles which results in end products with desired volume and uniform cellular structure. The functions of structured fat in batter and the corresponding properties imparted to the baked products can be listed as follows: (a) lubrication (tender texture); (b) aeration (volume and uniform cell structure); (c) retain long-term softness (shelf life), and (d) emulsify and hold water (prevent drying and retain moistness over storage). ${ }^{59}$

Since a significant amount of solid fat is used in preparation of bakery products, this product category would greatly benefit from substitution of solid fat with structured oil. Accordingly, exploring bakery applications of structured oil have received a great deal of attention from researchers working in the field of healthy structuring of foods. Most of these studies have dealt with the comparative evaluation of dough/batter properties (density, microstructure and rheology) and finished products characteristics (texture, stability and sensorial aspects). It has usually been found that the rheology of intermediate products (cake batter and cookie dough) prepared using structured oil is much less developed compared to the ones prepared using commercial bakery fats (shortening or baking margarine). However, the end baked products show comparable texture, stability and sensorial properties. ${ }^{60-64}$ The structured systems used in these studies were wax-based gels, hydrophilic polymer-based gels and MAG-based oil in water emulsion. Recent work reported by Blake and Marangoni ${ }^{65}$ suggests that structured oil systems could also find applications in laminated bakery products (puff pastry, croissant etc.) which are by far the most notorious category of products that poses the biggest challenge for fat substitution. New innovations in this product category will be of particular interest to the bakery industry world over.

\section{Margarines and spreads}

By definition, margarines are classified as fat continuous emulsions containing at least $80 \mathrm{wt} \%$ fat, spreads on the other hand, constitutes a broad range of products categorized based on the fat content as high-fat (70-82 wt\%), medium-fat (48-60 wt\%), low-fat (35-42 wt\%) and very low-fat $(<30 \mathrm{wt} \%)$ spreads. While, the low and very low-fat spreads are intended for 'spreading-on-bread' kind of applications, the high fat spreads are predominantly used for cooking/frying applications. In margarines and spreads, solid fat (or crystalline network of fat crystals) play three key roles: (i) provide structure (firmness, texture and spreadability); (ii) contribute to product stability (pickering stabilization of dispersed water droplets); and (iii) influence the sensorial properties (melt-in-mouth effect, emulsion destabilization and flavour release). These functions have to be kept in mind when substitution of solid fats with structured oil systems is considered.

For margarine and spreads applications, wax-based oleogels are considered to be the most suitable options. Hwang and coworkers evaluated the possibility of incorporating wax-based oleogels (prepared from sunflower wax, SW, Rice bran wax, RBW and Candelilla wax, CW) in margarine formulation. ${ }^{66}$ They found that the gelation efficiency of waxes in oil cannot be directly correlated to their effectiveness in margarine formation. For instance, CW formed firm oleogels, but the margarine formulation of $\mathrm{CW}$ was unstable and showed phase separation. Of the three waxes tested in this study, SFW was found to be the best structurant, forming stable margarine (at about $10 \mathrm{wt} \% \mathrm{SFW}$ ) with firmness comparable to commercial margarine. However, the melting point of wax-based margarine was comparably much higher than commercial margarine which is attributed to the high melting nature of wax. Waxbased oleogels (particularly, shellac wax oleogel) have also been explored for creating spreads with different fat contents (40-80 wt $\%){ }^{62}$ At low fat content, the stabilization of emulsion becomes more challenging because of the enhanced viscosity of the emulsion (resulting from the increase in the phase volume of dispersed water droplets) and a decrease in the crystalline fat phase resulting in larger droplets and increased possibility of their aggregation and coalescence. Thus, the choice of emulsifier as well as the selection of a high performance hard stock becomes more critical in formulating reducedand low-fat spreads. Surprisingly, it was found that shellac wax oleogel could be used as a fat phase to generate a range of emulsions at 80, 60, 50 and $40 \mathrm{wt} \%$ fat content without requiring any emulsifier or supplementary hard stock. ${ }^{62}$ The ability of shellac wax to stabilize these emulsions was attributed to the presence of a high proportion of fatty alcohols that helped 
in the initial dispersion of water droplets followed by interfacial and bulk crystallization of wax particles. ${ }^{19}$ It should be noted however that these were coarse emulsions with droplet size ranging from $25-40 \mu \mathrm{m}$. In case of non-wax-based systems (oleogels made using polymers and self-assembled structures), water incorporation usually results in a structure collapse unless the structure is supplemented by a small amount of solid fat to create what is called as 'hybrid systems' i.e. structured system created by exploiting both oleogelation and fat crystallization principles.

\section{Meat products}

The use of structured oil systems in reformulated meat products has been recently reviewed comprehensively by JimenezColmenero et $a l^{49}$ Since, improvement of fatty acid profile (higher proportion of mono and poly unsaturated fatty acids) is one of the main goals for reformulating meat products, structured oil systems could offer a lot of possibilities. Systems such as oleogels, structured emulsions and oil bulking systems have been explored for formulation and stabilization of fresh, cooked and fermented meat products such as meat suspensions and sauces, ${ }^{67,68}$ meat batters, ${ }^{69}$ sausages, ${ }^{70,71}$ patties $^{72}$ and frankfurters. ${ }^{73,74}$

The two aspects that are considered when using fat replacers in meat products are: reduction in total fat content (and calories) and improvement in fatty acid profile. The former is mainly achieved by replacing a substantial part of animal fat in meat products with structured emulsion systems consisting of liquid oil and gelled water, while in the latter case, liquid oil gelled using structuring agents is used as fat replacer. In addition, oleogelation (using lipid additives such as MAGs and lecithin) has also been used solely for stabilization of meat suspensions and sauces (which are basically oil-based suspensions) without significantly altering the fatty acid profile or reduction in fat content. ${ }^{67,68}$ In case of cooked products, it is important that the fat replacers (like polymer oleogels, structured emulsion and oil bulking systems) are able to replicate functionalities related to water and fat binding (to provide juiciness and prevent fat-loss during cooking), heat transfer and lipid oxidation (prevent rancidity).

\section{Chocolate applications}

There are only a handful of studies demonstrating the use of oil structuring approaches for chocolate applications, these studies include: development of heat resistant chocolates, complete replacement of oil binder in chocolate paste, partial replacement of palm oil in praline fillings and controlling the oil migration-induced fat bloom. The latter two examples refers to recent unpublished work from our group.

Ordinary chocolate can be considered as a dispersion of non-fat solid particles (cocoa and sugar particles, proteins, fibres etc.) in fat continuous phase (cocoa butter). The melting properties of chocolate is therefore linked to the physical state of cocoa butter which tends to soften at high temperatures encountered in the tropical regions. Formulating chocolates that can withstand high temperatures while still retaining appreciable melt-in-mouth properties is an unresolved challenge. Stortz and co-workers studied the possibility of using structuring properties of EC to develop chocolates that can retain their shape even at temperatures above $40{ }^{\circ} \mathrm{C} .{ }^{75} \mathrm{EC}$ was dispersed in molten chocolate through solvent substitution method by first mixing the ethanol solution of EC with molten chocolate followed by solvent evaporation to trigger in situ oleogel formation of EC in the fat phase. The interaction and subsequent oil trapping network formation of EC with sucrose particles contributed to heat resistance properties of chocolates. ${ }^{76}$

Chocolate pastes are dispersions of cacao powder and sugar particles in oil continuous medium structured with considerable amount of crystalline fat phase. Rheologically, a chocolate paste behaves like an arrested 'soft' solid at rest (to prevent sedimentation of dispersed particles as well as the separation of liquid oil) while displaying a more liquid-like spreading behaviour (yielding) under applied force. ${ }^{62}$ This viscoelastic behaviour of chocolate paste is imparted by incorporation of a significant amount ( $>20 \mathrm{wt} \%$ ) of solid fats (hydrogenated oils or natural oils with high levels of saturated fatty acids such as palm oil) in the formulation. Additionally, specialized ingredient such as an oil binder (fully hardened vegetable fat) is added to the formulation to prevent 'oiling out' during storage. Together, these ingredients contribute to the total saturated fat content of chocolate pastes. Based on the studies performed in our lab, we found that it was possible to replicate the functionality of oil binder with a structuring agent. ${ }^{62}$ For our study we used shellac wax at a concentration of $1.5 \mathrm{wt} \%$ for complete replacement of oil binder. Since shellac was added in form of an oleogel, we could also manage to partially replace palm oil $(\approx 27 \%)$ with more healthier rapeseed oil. These reformulated paste showed no sign of 'oiling out' when subjected to accelerated stability tests.

Oil migration is a major issue in filled confectionary products such as pralines (which usually consists of a hard chocolate shell with a softer filling). Liquid oil migrating from the softer core to the outer surface act as a solvent and solubilizes some high melting TAG crystals of cocoa butter. These crystals can then recrystallize into large crystals on the surface. Because of their bigger dimensions, these crystals scatter light resulting in a dull-white appearance as seen in Fig. 6. Oleogelation has been proposed as an option to control fat-bloom by restricting the mobility of oil in such systems. ${ }^{58}$ Currently, we have evaluated the possibility of using wax-based oleogels to restrict the fat-bloom formation while replacing a part of palm oil in the filling formulation at the same time. Based on our initial results we have found that the rheological properties of reference filling can be replicated even at $50 \%$ replacement of palm oil with oleogels made from only 2-2.5 wt\% of beeswax in rice bran oil. The fat-bloom studies of these samples are currently in progress.

\section{Conclusions and future trends}

Even though the field of oil structuring is in its infancy, the initial reports in this area have generated a wide spread inter- 


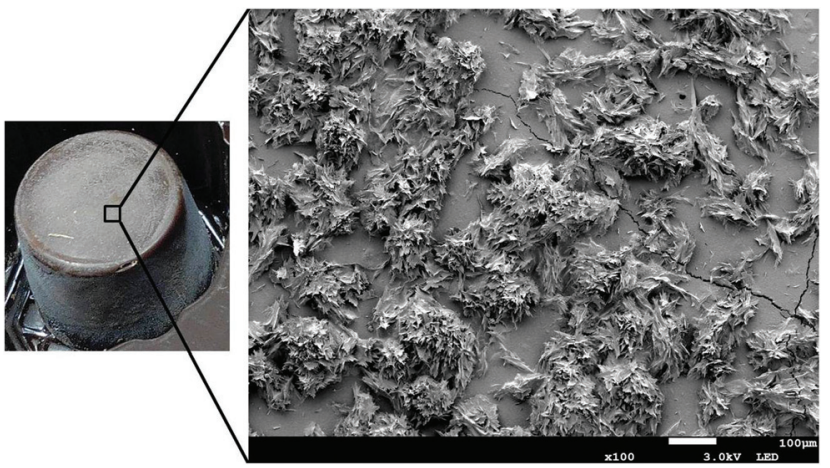

Fig. 6 Photograph of a praline sample showing fat-bloom. The cryoSEM image confirms the presence of large crystals, these crystals are responsible for the scattering of light leading to a dull-white appearance of the surface.

est from academia and industry alike. Especially in the last two years, we have seen a sudden rise in different forms of communication (journal articles, popular press and dedicated sessions at scientific conferences) related to this field. Fuelled by the real industrial needs, the field has been hailed as the possible answer to the unresolved challenge of saturated fat reduction in food products.

As reviewed in this paper, so far many different approaches of oil structuring have been explored, ranging from gelation of oil phase by unconventional additives to smart structuring of biphasic systems with the help of suitable hydrocolloids. In case of oil gelation, even though a number of structurants have been identified, there is still a need to find a food-grade oleogelator that is economical, efficient at low concentration, tolerant to processing conditions and compatible with the product matrix. It should be noted that fields such as pharmaceuticals (dermal drug delivery systems) and cosmetics have already been using the principles of oleogelation for a long time, referring to some of the studies from these fields may help food researchers to develop cross-disciplinary ideas to identify new structurants for food applications.

Among the food-grade oleogelators explored so far, natural waxes comes closest to matching all the desired characteristics. However, as they are only approved as indirect additives, there are regulatory concerns which needs to be addressed. Also, due to the susceptibility of waxes to post crystallization changes (crystal aggregation), wax-based oleogels may not be stable over long storage periods.

Polymers such as cellulose derivatives, proteins and other hydrophilic polysaccharides could also be considered as ideal structurants, however, additional processing steps required for oleogelation (such as high temperature treatment, lyophilisation, solvent removal etc.) makes polymer-based oleogels less feasible for scale-up. Although, it should be noted that some polymer-based oleogels have excellent long-term stability.

Exploring synergistic interactions among known food-grade ingredients is also a popular approach in the field of oleogelation. However, most of these synergistic combinations produce weak gels which may not be able to provide the desired functionality in real food systems. In some cases, it is advisable to incorporate a small amount of crystalline phase (to develop 'hybrid systems') as it might be too overambitious to rely entirely on the structurants (which are used at very low concentrations) to provide the desired firmness/hardness.

In contrast to oleogels, structured biphasic systems offer much more flexibility in terms of the choice of approved additives (hydrocolloids) that can be used. Moreover, in addition to decreasing the saturated fat content, these systems also contribute to overall fat (calorie) reduction. Rather than looking for specific structurants, these approaches rely more on smart dispersion technologies. However, these systems are very specific for the product types and there is no 'one size fits all' solution. In addition, since these systems rely heavily on structuring of the water phase rather than the oil phase, the likelihood of matching the functionality of references (in terms of texture and mouth feel) is relatively low.

Irrespective of the approach used, the created structured oil systems can serve as solid fat replacers in both water-free (shortenings, chocolates and chocolate pastes) and watercontaining (cooked meat products, margarine and spreads) products (refer Fig. 7 for some examples). Some of the important functionalities imparted by structured oil systems include texture and structure build-up, stabilization of dispersions and oil immobilization/oil binding.

The field of edible oil structuring has seen a lot of progress in last few years and going by the current trend, more detailed studies on oil structuring and its application in real food systems will emerge in the coming years. Special efforts need to be reserved for investigating the compatibility of structured systems with the product matrix and the sensorial aspects of structured oil containing food products.

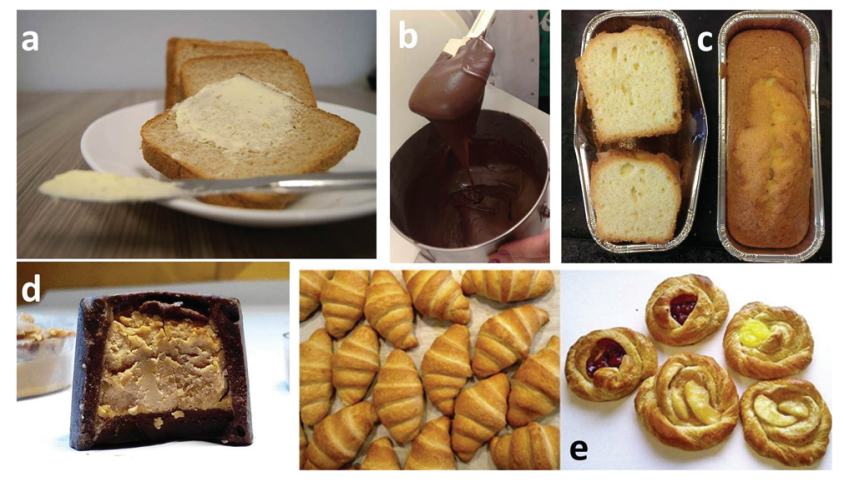

Fig. 7 Photographs of different food products prepared using structured oil: (a) Emulsifier-free, low-fat spread prepared using natural wax as structurant; (b) chocolate paste where oil binder is replaced with shellac wax; (c) standard 4/4 cake prepared using gelled emulsion as a shortening alternative; (d) praline with hazelnut filling where $50 \mathrm{wt} \%$ palm oil is replaced with beeswax oleogel in rice bran oil and (e)* croissants (left) and danishes (right) prepared with structured emulsion (Coasun Laminate). ${ }^{65}$ * Reproduced with permission from Elsevier Ltd. 


\section{Acknowledgements}

This work is supported by Marie Curie Career Integration Grant (Project: SAT-FAT-FREE) within the $7^{\text {th }}$ European Community Framework Programme.

\section{References}

1 S. Stender, A. Astrup and J. Dyerberg, BMJ Open, 2012, 2.

2 S. Stender, A. Astrup and J. Dyerberg, BMJ Open, 2014, 4.

3 R. Chowdhury, S. Warnakula, et al., Ann. Intern. Med., 2014, 160, 398-406.

4 J. Daniel and R. Rajasekharan, J. Am. Oil Chem. Soc., 2003, 80, 417-421.

5 N. K. O. Ojijo, E. Kesselman, V. Shuster, S. Eichler, S. Eger, I. Neeman and E. Shimoni, Food Res. Int., 2004, 37, 385393.

6 N. K. O. Ojijo, I. Neeman, S. Eger and E. Shimoni, J. Sci. Food Agric., 2004, 84, 1585-1593.

7 A. Wright and A. Marangoni, J. Am. Oil Chem. Soc., 2006, 83, 497-503.

8 J. F. Toro-Vazquez, J. A. Morales-Rueda, E. DibildoxAlvarado, M. Charó-Alonso, M. Alonzo-Macias and M. M. González-Chávez, J. Am. Oil Chem. Soc., 2007, 84, 989-1000.

9 F. Gandolfo, A. Bot and E. Flöter, J. Am. Oil Chem. Soc., 2004, 81, 1-6.

10 A. Bot and W. M. Agterof, J. Am. Oil Chem. Soc., 2006, 83, 513-521.

11 M. Pernetti, K. van Malssen, D. Kalnin and E. Flöter, Food Hydrocolloids, 2007, 21, 855-861.

12 M. A. Rogers, A. J. Wright and A. G. Marangoni, Soft Matter, 2009, 5, 1594-1596.

13 T. Laredo, S. Barbut and A. G. Marangoni, Soft Matter, 2011, 7, 2734-2743.

14 A. I. Romoscanu and R. Mezzenga, Langmuir, 2006, 22, $7812-7818$.

15 A. R. Patel, N. Cludts, M. D. Bin Sintang, B. Lewille, A. Lesaffer and K. Dewettinck, ChemPhysChem, 2014, 15, 3435-3439.

16 A. R. Patel, P. S. Rajarethinem, N. Cludts, B. Lewille, W. H. De Vos, A. Lesaffer and K. Dewettinck, Langmuir, 2015, 31, 2065-2073.

17 A. R. Patel, D. Schatteman, A. Lesaffer and K. Dewettinck, RSC Adv., 2013, 3, 22900-22903.

18 A. R. Patel, D. Schatteman, W. H. D. Vos and K. Dewettinck, RSC Adv., 2013, 3, 5324-5327.

19 A. R. Patel, D. Schatteman, W. H. De Vos, A. Lesaffer and K. Dewettinck, J. Colloid Interface Sci., 2013, 411, 114-121.

20 A. R. Patel, B. Mankoc, M. D. Bin Sintang, A. Lesaffer and K. Dewettinck, RSC Adv. , 2015, 5, 9703-9708.

21 A. Patel, in Alternative Routes to Oil Structuring, Springer International Publishing, 2015, ch. 1, pp. 1-14.

22 A. Vintiloiu and J.-C. Leroux, J. Controlled Release, 2008, 125, 179-192.
23 E. D. Co and A. G. Marangoni, J. Am. Oil Chem. Soc., 2012, 89, 749-780.

24 L. S. K. Dassanayake, D. R. Kodali and S. Ueno, Curr. Opin. Colloid Interface Sci., 2011, 16, 432-439.

25 M. Pernetti, K. F. van Malssen, E. Flöter and A. Bot, Curr. Opin. Colloid Interface Sci., 2007, 12, 221-231.

26 A. G. Marangoni and N. Garti, in Edible Oleogels: Structure and Health Implications, ed. A. G. Marangoni and N. Garti, AOCS Press, Urbana, IL, USA, 2011, pp. 1-18.

27 L. Han, L. Li, B. Li, L. Zhao, G.-q. Liu, X. Liu and X. Wang, J. Am. Oil Chem. Soc., 2014, 91, 1783-1792.

28 A. R. Patel, M. Babaahmadi, A. Lesaffer and K. Dewettinck, J. Agric. Food Chem., 2015, 63, 4862-4869.

29 C. Doan, D. Van de Walle, K. Dewettinck and A. Patel, J. Am. Oil Chem. Soc., 2015, 92, 801-811.

30 A. Bot and E. Floter, in Edible Oleogels: Structure and Health Implications, ed. A. G. Marangoni and N. Garti, AOCS Press, Urbana, IL, USA, 2011, pp. 49-79.

31 C. V. Nikiforidis and E. Scholten, RSC Adv., 2014, 4, 24662473.

32 M. Davidovich-Pinhas, S. Barbut and A. G. Marangoni, Carbohydr. Polym., 2015, 117, 869-878.

33 M. Davidovich-Pinhas, A. J. Gravelle, S. Barbut and A. G. Marangoni, Food Hydrocolloids, 2015, 46, 76-83.

34 C. V. Nikiforidis and E. Scholten, RSC Adv., 2015, 5, 3778937799.

35 Y. Huang, M. He, A. Lu, W. Zhou, S. D. Stoyanov, E. G. Pelan and L. Zhang, Langmuir, 2015, 31, 1641-1648.

36 J. M. Aiache, P. Gauthier and S. Aiache, Int. J. Cosmet. Sci., 1992, 14, 228-234.

37 P. Gauthier, S. Aiache and J. M. Aiache, Int. J. Cosmet. Sci., 1996, 18, 229-235.

38 H. Adelmann, B. P. Binks and R. Mezzenga, Langmuir, 2012, 28, 1694-1697.

39 R. Mezzenga and S. Ulrich, Langmuir, 2010, 26, 1665816661.

40 C. P. Whitby and A. J. Onnink, Adv. Powder Technol., 2014, 25, 1185-1189.

41 Z.-M. Gao, X.-Q. Yang, N.-N. Wu, L.-J. Wang, J.-M. Wang, J. Guo and S.-W. Yin, J. Agric. Food Chem., 2014, 62, 26722678.

42 F. Rouquerol, J. Rouquerol and K. Sing, in Adsorption by Powders and Porous Solids, ed. F. Rouquerol, J. Rouquerol and K. Sing, Academic Press, London, 1999, pp. 1-26.

43 A. R. Patel and K. Dewettinck, European Journal of Lipid Science and Technology, 2015, DOI: 10.1002/ejlt.201400553.

44 A. R. Patel, in Alternative Routes to Oil Structuring, Springer International Publishing, 2015, ch. 3, pp. 29-39.

45 N. W. Higgins and R. L. Daniels, Eur. Pat., 2568818 A1, 2011. 46 S. Metin, P. R. Smith, S. Veelaert, P. Moran and D. M. A. Karleskind, US Pat., 20150064329 A1, 2013.

47 S. M. Clegg, A. K. Moore and S. A. Jones, J. Food Sci., 1996, 61, 1073-1079.

48 A. K. Zetzl and A. G. Marangoni, in Edible Oleogels: Structure and Health Implications, ed. A. G. Marangoni and N. Garti, AOCS Press, Urbana, IL, USA, 2011, pp. 19-47. 
49 F. Jimenez-Colmenero, L. Salcedo-Sandoval, R. Bou, S. Cofrades, A. M. Herrero and C. Ruiz-Capillas, Trends Food Sci. Technol., 2015, 44, 177-188.

50 A. G. Marangoni, S. H. J. Idziak, C. Vega, H. Batte, M. Ollivon, P. S. Jantzi and J. W. E. Rush, Soft Matter, 2007, 3, 183-187.

51 H. D. Batte, A. J. Wright, J. W. Rush, S. H. J. Idziak and A. G. Marangoni, Food Res. Int., 2007, 40, 982-988.

52 L. Salcedo-Sandoval, S. Cofrades, C. Ruiz-Capillas, J. Carballo and F. Jiménez-Colmenero, Meat Sci., 2015, 101, 95-102.

53 A. M. Herrero, C. Ruiz-Capillas, F. Jiménez-Colmenero and P. Carmona, J. Agric. Food Chem., 2014, 62, 59635971.

54 C. Speirs, in New Food, Russell Publishign Limited, Kent, UK, 2014.

55 A. R. Patel, Y. Rodriguez, A. Lesaffer and K. Dewettinck, RSC Adv., 2014, 4, 18136-18140.

56 A. M. Jensen, Palsgaard Technical Paper-How to successfully manufacture a low fat margarine spread with only $10 \%$ fat, 2013.

57 A. R. Patel, in Alternative Routes to Oil Structuring, Springer International Publishing, 2015, ch. 5, pp. 51-62.

58 N. E. Hughes, A. G. Marangoni, A. J. Wright, M. A. Rogers and J. W. E. Rush, Trends Food Sci. Technol., 2009, 20, 470480.

59 B. S. Ghotra, S. D. Dyal and S. S. Narine, Food Res. Int., 2002, 35, 1015-1048.

60 A. Goldstein and K. Seetharaman, Food Res. Int., 2011, 44, 1476-1481.

61 A. R. Patel, N. Cludts, M. D. B. Sintang, A. Lesaffer and K. Dewettinck, Food Funct., 2014, 5, 2833-2841.
62 A. R. Patel, P. S. Rajarethinem, A. Gredowska, O. Turhan, A. Lesaffer, W. H. De Vos, D. Van de Walle and K. Dewettinck, Food Funct., 2014, 5, 645-652.

63 E. Ylmaz and M. Ogutcu, Food Funct., 2015, 6, 1194-1204.

64 A. Jang, W. Bae, H.-S. Hwang, H. G. Lee and S. Lee, Food Chem., 2015, 187, 525-529.

65 A. I. Blake and A. G. Marangoni, Food Res. Int., 2015, 74, 284-293.

66 H.-S. Hwang, M. Singh, E. Bakota, J. Winkler-Moser, S. Kim and S. Liu, J. Am. Oil Chem. Soc., 2013, 90, 1705-1712.

67 F. R. Lupi, D. Gabriele, L. Seta, N. Baldino and B. de Cindio, Eur. J. Lipid Sci. Technol., 2014, 116, 1734-1744.

68 F. R. Lupi, D. Gabriele, N. Baldino, L. Seta, B. de Cindio and C. De Rose, Eur. J. Lipid Sci. Technol., 2012, 114, 13811389.

69 C. Ruiz-Capillas, P. Carmona, F. Jiménez-Colmenero and A. M. Herrero, Food Chem., 2013, 141, 3688-3694.

70 C. Poyato, D. Ansorena, I. Berasategi, Í. Navarro-Blasco and I. Astiasarán, Meat Sci., 2014, 98, 615-621.

71 F. Jiménez-Colmenero, M. Triki, A. M. Herrero, L. Rodríguez-Salas and C. Ruiz-Capillas, LWT - Food Sci. Technol., 2013, 51, 158-163.

72 L. Salcedo-Sandoval, S. Cofrades, C. Ruiz-Capillas and F. Jiménez-Colmenero, Meat Sci., 2014, 98, 795-803.

73 F. Jiménez-Colmenero, A. Herrero, T. Pintado, M. T. Solas and C. Ruiz-Capillas, Food Res. Int., 2010, 43, 2068-2076.

74 A. K. Zetzl, A. G. Marangoni and S. Barbut, Food Funct., 2012, 3, 327-337.

75 T. A. Stortz and A. G. Marangoni, Food Res. Int., 2013, 51, 797-803.

76 T. A. Stortz, D. C. De Moura, T. Laredo and A. G. Marangoni, RSC Adv., 2014, 4, 55048-55061. 